\title{
Effect of calmodulin on the stimulation of capacitation and acrosome reaction of frozen thawed bull spermatozoa
}

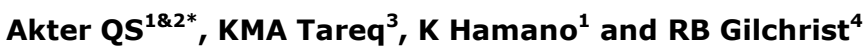

\begin{abstract}
${ }^{1}$ Laboratory of Reproductive Functions in Mammals, Faculty of Agriculture, Shinshu University, Minamiminowamura, Nagano-ken 399-4598, Japan; '2Department of Genetics and Animal Breeding, Faculty of Animal Science and Veterinary Medicine, Patuakhali Science and Technology University, Barisal-8210, Bangladesh; ${ }^{3}$ Faculty of Animal Science and Veterinary Medicine, Patuakhali Science and Technology University, Barisal-8210, Bangladesh; ${ }^{4}$ Oocyte Biology Research Unit, Discipline of Obstetrics \& Gynaecology, School of Women's \& Children's Health, The University of New South Wales, Sydney, NSW 2052, Australia
\end{abstract}

\begin{abstract}
Capacitation and acrosome reaction (AR) are the prerequisites for successful fertilization by mammalian spermatozoa. Intracellular calcium $\left(\mathrm{Ca}^{2+}\right)$ has a regulatory role in sperm motility, capacitation, and AR. Calmodulin (CaM) antagonists calmidazolium (CZ) and N-(6-aminohexyl)-5-chloro-1naphthalenesulfonamide (W7) were used to investigate the possible role of $\mathrm{CaM}$, a $\mathrm{Ca}^{2+}$ specific binding protein, on motility, capacitation and AR of frozen-thawed bovine spermatozoa. Capacitation and AR in sperm were evaluated by using chlortetracycline (CTC) staining technique. Addition of the $1 \mathrm{mM}$ dibutyryl cAMP (dbcAMP) and $100 \mu \mathrm{M}$ 1-methy 1-3-isobutylxanthine (IBMX) to CaM antagonists treated sperm incubated in the presence of $\mathrm{NaHCO}_{3}$ and $\mathrm{CaCl}_{2}$ in media overcome the inhibitory effects of these antagonists to support capacitation and $A R$ at $4 \mathrm{~h}$ of incubation period. In contrast, addition of dbcAMP with IBMX induced $A R$ in spermatozoa incubated with $\mathrm{NaHCO}_{3}$-free medium but these compounds did not induce $A R$ in cells incubated in $\mathrm{CaCl}_{2}$-free medium. However, the addition of dbcAMP and IBMX partially, but significantly $(p<0.01)$ reversed the inhibitory effect of W7 and $C Z$ on the sperm capacitation and AR. These results suggest that CaM may play an important role in the regulation of capacitation and AR in frozen-thawed bovine spermatozoa.
\end{abstract}

Key words: acrosome reaction, frozen thawed bull spermatozoa, capacitation, calmodulin

Bangladesh Animal Husbandry Association. All rights reserved.

Bang. J. Anim. Sci. 2016. 45 (3): 1-9

\section{Introduction}

Artificial insemination is the most widely applied tool in modern animal breeding and thereby facilitating extensive utilization of cryopreserved spermatozoa. Mammalian spermatozoa undergo a variety of physiological and biochemical modifications during their transit through female reproductive tract (Harayama, 2013). These changes are collectively termed "capacitation" and regulated by sperm interaction with female reproductive tract (Kuo et al., 2016). However, sperm capacitation is associated with $\mathrm{Ca}^{2+}$ uptake (Handrow et al., 1989), decreased binding of proteins to calmodulin (CaM) (Leclerc et al., 1992), increased cyclic adenosine mono phosphate CAMP (White and Aitken, 1989), activation of CAMP dependent protein kinase-A (Visconti et al., 1997), and changes in tyrosine phosphorylation levels (Tardif et al., 2001). But, trans-membrane and intracellular signaling event regulating sperm capacitation are likewise, poorly understood.

In vitro capacitation and induction of $A R$ can be accomplished using several treatments (Tanphaichitr and Hansen, 1994). After capacitation, one primary signal that initiates the $A R$ is a change in sperm plasma membrane permeability for $\mathrm{Ca}^{+}$-influx. Therefore, divalent cationic ionophores, such as A23187, artificially induce calcium entry into sperm, resulting in the AR in bovine (Triana et al., 1980) and stallion (Farlin et al., 1992) spermatozoa. However, it is not clear how sperm functions are affected by these media constituents. The onset of both capacitation and $A R$ are dependent on the presence of bovine serum albumin (BSA), $\mathrm{Ca}^{2+}$ and $\mathrm{NaHCO}_{3}$ in the culture medium, three components that have been demonstrated to be essential for in vitro capacitation of sperm from a variety of different mammals (Yanagimachi et al.,

\section{*Corresponding author: akter_shrmn@yahoo.com}


1994). The underlying $\mathrm{Ca}^{2+}$-regulated pathways involved in the initiation and maintenance of sperm motility have not yet been fully understood. $\mathrm{Ca}^{2+}$ can directly bind to membrane phospholipids and to several enzymes, with the subsequent modification of membrane properties and enzymatic activity. This cation may also act through binding to $\mathrm{CaM}$. The $\mathrm{Ca}^{2+}$-binding protein CaM in somatic cells modulates the activity of key enzymes also identified in mammalian sperm cells, including adenylyl cyclases (Mons et al., 1999), phosphatases (Rusnak and Mertz, 2000), phosphodiesterases (Wasco and Orr, 1984) and protein kinases (Hook and Means, 2001). Furthermore, immunocytochemical studies reveal that CaM is present in the head and flagellum of mammalian sperm (Moriya et al., 1993), suggesting that CaM could be involved in functions occurring in the sperm head, tail, or both locations.

Moreover, CaM operates on various targets in sperm; the roles of CaM in sperm physiology are diverse and include the support of capacitationrelated changes in plasma membrane properties, modulation of CAMP levels (Fournier et al., 2003), and the ability to undergo the AR (Bendahmane et al., 2001). Cyclic AMP is implicated in many sperm functions such as capacitation (Visconti et al., 1995), AR (Kopf and Gerton, 1991) and initiation and maintenance of motility (Yanagimachi et al., 1994). Recently, Zeng and Tulsiani et al.,(2003) demonstrated that elevated intracellular $\mathrm{Ca}^{2+}$ is required for the initiation of hyperactivated motility and capacitation suggesting that $\mathrm{CaM}$ and $\mathrm{Ca} / \mathrm{CaM}$-stimulated pathways are involved. Although, there are several studies that have shown the importance of $\mathrm{CaM}$ and $\mathrm{Ca} / \mathrm{CaM}$ levels in sperm function, direct influence of CaM antagonists on capacitation and AR in bovine spermatozoa have not yet been definitively demonstrated.

In this study, we have investigated the role of CaM antagonists to understand its possible significance in multiple aspects of frozen-thawed bovine sperm capacitation and AR. Therefore, from these points of view the present study was designed to know the effect of several components including calcium ionophore, $\mathrm{CaCl}_{2}$, $\mathrm{NaHCO}_{3}$ and CAMP agonists on bovine sperm capacitation and AR.

\section{Materials and Methods}

\section{Chemicals and media}

All chemicals used in this experiment were purchased from Sigma Chemicals (St. Louis, MO) with the following exceptions: dimethyl-sulfoxide (DMSO), bovine serum albumin, glutaraldehyde, sodium pyruvate were obtained from Wako Pure Chemicals Industries and L-cysteine was obtained from Kanto Chemical Co., Inc, Japan. All chemicals were the highest analytical grade. Stock solutions of calmidazolium (CZ) and $\mathrm{N}$-(6aminohexyl)-5-chloro1- naphthalenesulfonamide (W7) were prepared in $1.5 \%$ dimethyl sulfoxide (DMSO) and stored at $-20^{\circ} \mathrm{C}$. The basic medium used throughout these experiments was a modified Bracket and Oliphant (BO; 1975) for semen dilution and washing.

\section{Preparation of semen samples}

Frozen semen of Holstein Friesian bull was obtained from the Nagano Livestock Improvement Association of Japan. The same pool of semen was used throughout this study. Frozen straws were thawed in a water bath at $37^{\circ} \mathrm{C}$ for 10 to 14 seconds. The frozen-thawed semen samples were suspended in $B O$ media and washed thrice with BO-BSA through centrifugation (5 minutes at $2000 \mathrm{rpm}$ ) to remove the extra-cellular debris and egg-yolk particles. After centrifugation the supernatant was removed and then $0.5 \mathrm{ml}$ BO-BSA added. The sperm suspension was gently mixed and again centrifuged, same procedure was performed for thrice for washing of bovine sperm. The concentration of sperm was estimated using a haemocytometer and adjusted with BO-BSA to $50 \times 10^{6} \mathrm{sperm} / \mathrm{ml}$ and incubated at $38.5^{\circ} \mathrm{C}, 5 \%$ $\mathrm{CO}_{2}$ for $0-4 \mathrm{~h}$ incubation period.

\section{Capacitating and AR inducing agent preparation}

A solution of $0.5 \mu \mathrm{M}$ calcium ionophore was prepared by diluting ionophore $(1 \mathrm{mg})$ into $\mathrm{EtOH}$ DMSO $(955 \mu \mathrm{l})$. This solution was stored at $-20^{\circ} \mathrm{C}$ until use. A working solution of calcium ionophore ( $2 \mathrm{mM}$ ) was made by diluting the stock solution into BO-Caffeine $(1 \mathrm{ml})$. The stock of $1 \mathrm{mM}$ cAMP and $100 \mu \mathrm{M}$ IBMX were prepared by dissolving both CAMP and IBMX (0.005 g) in DMSO (15.18 
$\mathrm{ml}$ and $2.25 \mathrm{ml}$, respectively) and was stored at $20^{\circ} \mathrm{C}$ until use.

\section{Evaluation of motility}

After completion of the $0-4 \mathrm{~h}$ incubation period, the subsamples $(10 \mu \mathrm{L})$ of each sample were transferred to an examination chamber (Sekisui, Tokyo, Japan), placed on a warmer set (MP10DM; Kitazato, Tokyo, Japan) kept at $38^{\circ} \mathrm{C}$, and the motility was examined under a light microscope $(100 \times)$. Sperm motility was assessed by determining the percentage of spermatozoa in each of the following five categories of movement: (a) rapid progressive motility; (b) slow or sluggish progressive motility; (c) nonprogressive motility; (d) immotility and e) dead. Motility was then expressed as a progressive motility percentage. The percentage of spermatozoa with progressive motility was determined subjectively by scoring 400 individual sperm population in each sample at $0,1,2$ and 4 $h$ of incubations.

\section{Evaluation of viability}

Sperm viability was determined by Hoechst 33258 staining (Sigma, B-2883). Fifty-microliter sample was mixed with $1 \mu \mathrm{l}$ of modified Hoechst 33258 solution and sperm was incubated with the dye Hoechst 33258 for 3 minutes. After incubation, the slides were covered with $10 \mu$ of sperm suspension and mounted on a glass slide under a coverslip. At least 100 sperms per slide were counted by fluorescent microscopy (FLmicroscopy, $420 \mathrm{~nm}$ ) and spermatozoa were classified as dead when nuclei showed bright blue fluorescence over sperm head and live when sperm head was not strongly fluorescent.

\section{Evaluation of Capacitation and AR by the CTC Assay}

The fluorescent antibiotic CTC was used to assess sperm capacitation. We used a modified version of the CTC assay described by Ward and Storey (Ward and Storey, 1994). In addition to simultaneous evaluation of cell viability and capacitation state, CTC staining was coupled with Hoechst 33258 staining Storey (Ward and Storey, 1994). CTC $(750 \mu \mathrm{M})$ was prepared in $20 \mathrm{mM}$ tris buffer containing $130 \mathrm{mM} \mathrm{NaCl}$ and $5 \mathrm{mM} \mathrm{DL}-$ cysteine and the $\mathrm{pH}$ was adjusted to 7.8. At room temperature, $50 \mu \mathrm{l}$ of Hoechst stained sperm sample was mixed with $450 \mu \mathrm{l}$ of Polyvinylpyrrolidone-PBS. After 5 min centrifugation at $1500 \mathrm{rpm}$, the supernatant was discarded. Then $0.3 \%$ BO-BSA added and finally the sperm sample was mixed with $100 \mu \mathrm{l}$ CTC solution. After $30 \mathrm{sec}, 5 \mu \mathrm{l}$ of $12.5 \%$ glutaraldehyde ( $\mathrm{pH} 7.4)$, was added. Finally, $5 \mu \mathrm{l}$ of $0.22 \mathrm{M} \mathrm{1}$, 4-diaza-byciclo (2.2.2) octane (DABCO, Sigma, USA) in 50\% glycerol was added to retard fluorescence fading and $10 \mu \mathrm{l}$ of this suspension was placed on a clean slide. After mounting with a coverslip, the slides were stored in dark at $4^{\circ} \mathrm{C}$ until counting within $24 \mathrm{~h}$. The slides were assessed with microscope (Olympus BX40) equipped with epifluorescence optics; cells were observed under blue-violet illumination (excitation at $400-440 \mathrm{~nm}$ and emission at 470 $\mathrm{nm}$ ). In addition, we assessed the HO-positive sperm (dead sperm) in the same field, and thus, the percentage of each CTC pattern in the live sperm population was determined. One hundred spermatozoa per slide were counted and classified according to the CTC patterns: $F$, showing uniform fluorescence on the head, indicating incapacitated, acrosome intact spermatozoa; B, represented by a fluorescencefree band on the post acrosomal region, indicating capacitated, acrosome intact spermatozoa; and AR, represented by a uniformly fluorescence-free head and with a fluorescence band in the equatorial region, indicating $A R$ as shown in Figure 1.

\section{Experimental layout}

In experiment 1 , the sperms were treated with various concentrations of CaM antagonists, CZ (5, 10, $15 \mu \mathrm{M})$ and $\mathrm{W} 7(50,75,100 \mu \mathrm{M})$. Then sperms were incubated up to $4 \mathrm{~h}$ at $38^{\circ} \mathrm{C}, 5 \%$ $\mathrm{CO}_{2}$. The inhibitor treatments were added to the sperm suspension immediately before incubation. Control without inhibitors received an equivalent amount of DMSO. At $0,1,2$, and $4 \mathrm{~h}, 10 \mu \mathrm{l}$ aliquots were removed for evaluation of motility. Viability, capacitation and AR were assessed at 0 and $4 \mathrm{~h}$ of incubation.

In experiment 2 , the sperms were then treated in separate analyses with, IA and dibutyryl cAMP (dbcAMP) plus 3-isobutyl-1-methylxanthine (IBMX) in the absence (control) or presence of CaM antagonists ( $C Z$ and W7). The concentration of $\mathrm{CZ}(5 \mu \mathrm{M})$ and $\mathrm{W} 7(50 \mu \mathrm{M})$ was selected from 
the first experiment. The mixture was then incubated in conditions $\left(38^{\circ} \mathrm{C}, 5 \% \mathrm{CO}_{2}\right.$ in air) for $4 \mathrm{~h}$.

\section{Statistical analysis}

Results are presented as mean \pm SEM of the number of samples indicated in each experiment. To determine whether there were significant differences among treatments and between conditions of incubation, means were compared using two tailed ANOVA. Multiple comparisons were made using Tukey's test, differences were considered to be statistically significant when $\mathrm{P}<0.01$.

\section{Results}

Effects of calmidazolium ( $\mathrm{CZ}$; a) and $\mathrm{N}$-(6aminohexyl)-5-chloro-1 Naphthalenesulfonamide (W7; b) on the percentages of sperm progressive motility are shown in Figure $1(a, b)$. Progressive motility were decreased in the sperm treated by the concentrations of $\mathrm{CZ}(5-15 \mu \mathrm{M})$ and $\mathrm{W} 7(50-$ $100 \mu \mathrm{M}$ ) than that of the control sperm after $1 \mathrm{~h}$ of incubation. The mean percentage of motile sperm of $\mathrm{CZ}$ and $\mathrm{W} 7$ treated group was less than $20 \%$ at $4 \mathrm{~h}$ of incubation. However, the effect of CZ and W7 was more severe at higher concentrations compared with the control in all incubation periods.

CaM antagonists, both CZ and W7 inhibited the sperm capacitation ( $B$ pattern) and AR pattern in a concentration-dependent manner. $C Z$, at $10 \mu \mathrm{M}$ significantly suppressed the frequency of the $B$ pattern, and inhibition was even greater at higher concentrations, $15 \mu \mathrm{M} \mathrm{CZ}$ compared with the control (Figure 2, a). Another CaM antagonist, W7 also decreased the percentage of $B$ pattern sperm in a concentration-dependent manner, with maximal inhibition at 75-100 $\mu \mathrm{M}$, the results is shown in Figure 2, (a). In contrast of two CaM antagonists, W7 did not significantly affect the B pattern even at $50 \mu \mathrm{M}$. Sperm were incubated for $4 \mathrm{~h}$ in $\mathrm{BO}$ medium, the percentage of AR sperm to about $20-25 \%$ in all treated group. Sperm treated with $5 \mu \mathrm{M} \mathrm{CZ}$ and $50 \mu \mathrm{M}$ W7 showed similar percentages of AR compared with the control (Figure. 2, b). But at higher concentrations of $\mathrm{CZ}$ and W7 were significantly shown to inhibit the acrosome reaction.
In the concentration range of 5-15 $\mu \mathrm{M}$ for $\mathrm{CZ}$ and 50-100 $\mu \mathrm{M}$ for W7, none of these CaM antagonists showed significant inhibitory effect on sperm viability. The proportion of live and dead sperm was separated by Hoechst Staining. The viability was around $61.7 \pm 1.7 \%$ at $0 \mathrm{~h}$ of incubation and $52 \pm 1.1 \%$ at $4 \mathrm{~h}$ of incubation, in both control and all treated groups (data not shown).
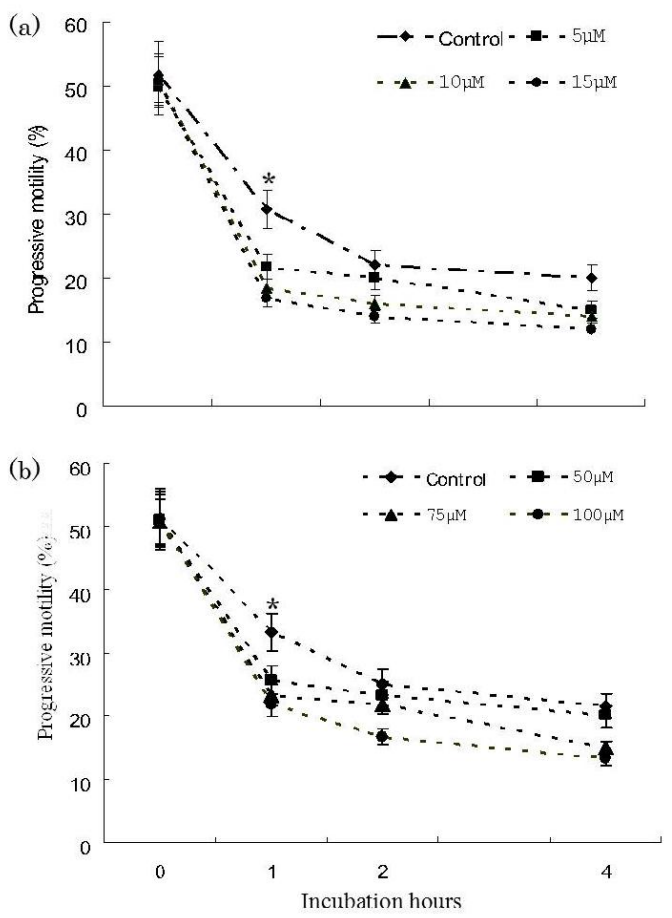

Figure 1. Effects of calmidazolium (CZ; a) and $\mathrm{N}$-(6aminohexyl)-5-chloro-1 Naphthalene sulfonamide (W7; b) on the percentages of sperm progressive motility. Spermatozoa incubated with various concentrations of $\mathrm{CZ}$, W7 and control sperm receiving no added antagonist. Sperm motility observed at $0,1,2$ and $4 \mathrm{~h}$ of incubation periods. Results are the mean \pm standard error $(n=3)$. * Significantly different $(P<0.01)$ from control.

When dbcAMP plus IBMX (a nonspecific inhibitor of CAMP and CGMP phosphodiesterases) was added to the incubation medium containing either $5 \mu \mathrm{M}$ or CZ $50 \mu \mathrm{M}$ W7 and incubated for $4 \mathrm{~h}$, the sperm displayed motility almost similar to that obtained when CAMP analogues were not added (data not shown). Thus, addition of cAMP analogues was unable to reverse the effects of $\mathrm{CaM}$ antagonists on motility. 

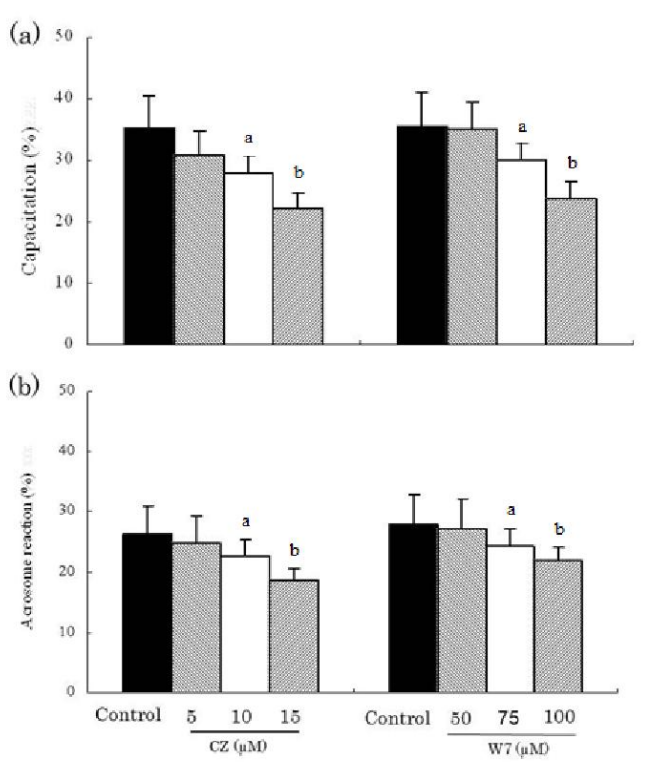

Figure 2. Effects of calmidazolium (CZ) and $\mathrm{N}$-(6aminohexyl)-5-chloro-1 naphthalene sulfonamide (W7) on the percentage of sperm showing capacitation (a) acrosome reaction (b) after CTC staining at $4 \mathrm{~h}$ of incubation periods with various concentrations of $\mathrm{CZ}$ and W7. Results are the mean \pm standard error $(n=3)$. a-b values with different letter superscripts are significantly different $(\mathrm{P}<0.01)$ from control.

The effect of cAMP-elevating agents (dbcAMP and IBMX) on sperm capacitation and AR of CaM inhibitors treated sperm samples containing with or without bicarbonate the results is shown in Figure $3(a, b)$. The capacitation pattern analysis (CTC/HO staining) revealed that the addition of dbcAMP and IBMX to samples in presence of bicarbonate produced a significant increase in sperm displaying CTC capacitation pattern (along with a decrease in not-capacitated pattern) relative to control samples at $4 \mathrm{~h}$ incubation, shown in Figure 3(a). CaM antagonists treated sperm incubated in medium devoid of $\mathrm{NaHCO}_{3}$ also did not display the CTC fluorescence B pattern. In contrast, the addition of $1 \mathrm{mM}$ dbcAMP plus $100 \mu \mathrm{M}$ IBMX to medium devoid of $\mathrm{NaHCO}_{3}$ could substitute for $\mathrm{NaHCO}_{3}$ in supporting the ability of the sperm to undergo the Zona pellucida-induced acrosome reaction (Figure $3 \mathrm{~b}$ ). In addition, in the presence of bicarbonate, a significantly higher value of the acrosome-reacted population was found in case both agents.
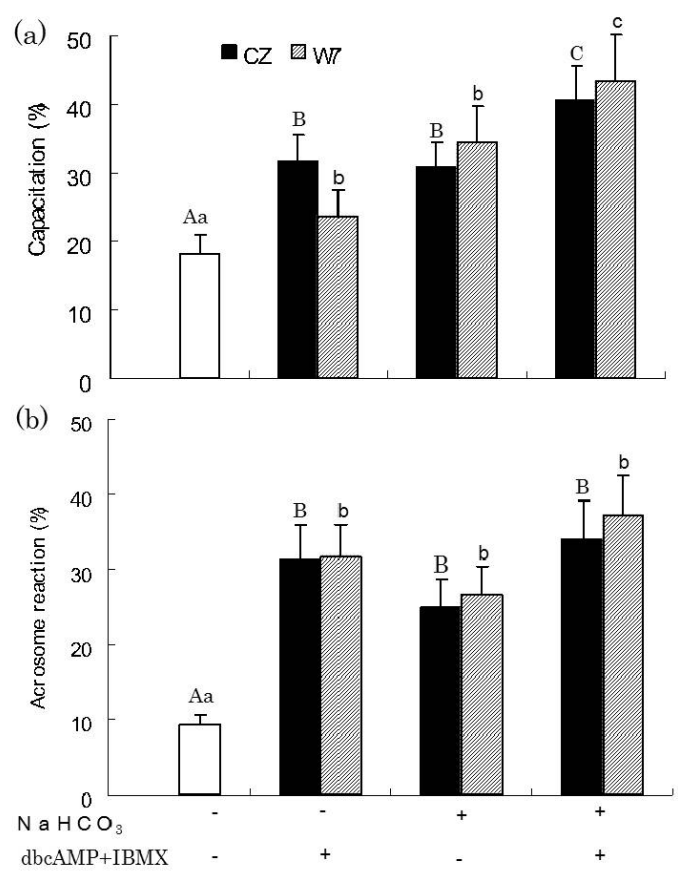

Figure 3. Effects of $1 \mathrm{mM}$ dibutyryl cAMP plus $100 \mu \mathrm{M}$ 3-isobutyl-1-methylxanthine (IBMX) in presence of $\mathrm{CZ}(5 \mu \mathrm{M})$ and $\mathrm{W} 7(50 \mu \mathrm{M})$ on the percentage of sperm showing (a) capacitation (B pattern) and (b) acrosome reacted (AR pattern) after chlortetracycline fluorescence (CTC) staining in media containing $\mathrm{NaHCO}_{3}$ or in medium devoid of $\mathrm{NaHCO}_{3}$ at $4 \mathrm{~h}$ of incubation period. Results are expressed mean \pm standard error $(n=3)$. a-c; A-B values with different letter superscripts are significantly different $(P<0.01)$ from each other.

The percentage of B and AR pattern significantly increased when CaM treated spermatozoa supplemented with dbcAMP plus IBMX in presence of $\mathrm{Ca}^{2+}$. When $5 \mu \mathrm{M} \mathrm{CZ}$ and $50 \mu \mathrm{M}$ W7 treated sperm were incubated for $4 \mathrm{~h}$ with $1 \mathrm{mM}$ dbcAMP and $100 \mu \mathrm{M}$ IBMX in presence of $\mathrm{CaCl}_{2}$ about $40-45 \%$ of the sperm displayed the $B$ pattern (Figure 4.a). The combination of dbcAMP and IBMX increased the percentage of acrosomereacted spermatozoa to about $35 \%$ in the presence of calcium in $5 \mu \mathrm{M} \mathrm{CZ}$ treated sperm. Similar results were observed when the spontaneous acrosome reaction was inhibited by W7, about $38 \%$ AR observed after treated with $\mathrm{dbcAMP}$ at $4 \mathrm{~h}$ of incubation (Figure $4 . \mathrm{b}$ ). The 
addition of $1 \mathrm{mM}$ dbcAMP plus $100 \mu \mathrm{M}$ IBMX to medium devoid of $\mathrm{CaCl}_{2}$ could not substitute for $\mathrm{CaCl}_{2}$ in supporting the ability of the sperm to display the CTC fluorescence B pattern in both CZ and W7 treated groups.

The calcium ionophore has been shown to accelerate the spontaneous AR. In order to investigate whether $A 23187$ can induce the $A R$, CaM treated spermatozoa were supplemented with $0.5 \mu \mathrm{M}$ IA. When sperm were incubated for 4 h with $5 \mu \mathrm{M} \mathrm{CZ}$ and calcium ionophore about $35 \%$ of the sperm displayed the AR pattern (Figure 5 b). However, when sperm were incubated with $50 \mu \mathrm{M}$ W7 and ionophore, the level of sperm with the AR pattern was increased from that of sperm incubated in $\mathrm{W} 7$ alone. In case of capacitation state, similarly an increased rate of $\mathrm{B}$ pattern was also observed with these groups; the result is shown Figure $5 \mathrm{a}$. The IA treated sperm did not alter the inhibition of $\mathrm{CZ}$ and W7 on sperm motility. The percentage of motile sperm was reduced significantly to less than $20 \%$ of motile cells when both CZ were incubated with IA at $4 \mathrm{~h}$ incubation (data not shown).

\section{Discussion}

The roles of CaM in sperm physiology are diverse and include the support of capacitation-related changes in plasma membrane properties (Bendahmane et al., 2001), modulation of cAMP levels (Fournier et al., 2003), and the ability to undergo the AR (Fournier et al., 2003). To identify the targets of $\mathrm{Ca}^{2+} / \mathrm{CaM}$ in sperm motility, capacitation and AR, we examined CaM antagonists ( $C Z$ and W7) for their ability to block CaM-dependent capacitation. In our study; both CZ and W7 depressed motility relative to that of control sperm. W7, a CaM antagonist was previously shown to reduce the proportion of hyperactivation among intact epididymal mouse sperm (Si and Olds-Clarke, 2000). These findings indicate that CaM plays an essential role in activating bovine sperm motility.

This study shows that inhibition of CaM inhibitors, CZ and W7 decreased capacitation and AR of bovine sperm, reported by the CTC assay. The B pattern after CTC staining was used as a probe for monitoring capacitation-dependent sperm membrane changes, since it is correlated with the sperm's ability to undergo the ZP-induced AR (Ward and Storey, 1994). Both W7 and CZ inhibited the $B$ pattern in a concentrationdependent manner (is shown in Figure 3). Furthermore, both $C Z$ and W7 inhibited the sperm's ability to undergo the AR.

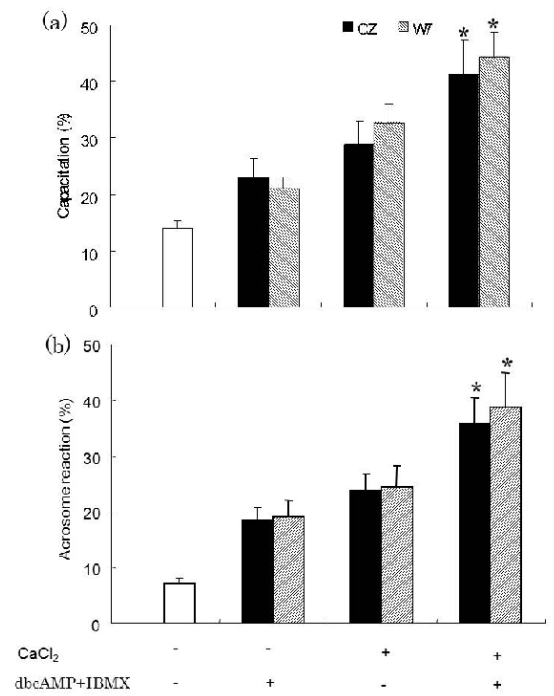

Figure 4. Effects of $1 \mathrm{mM}$ dibutyryl cAMP plus 100 $\mu \mathrm{M}$ 3-isobutyl-1-methylxanthine (IBMX) in presence of $C Z(5 \mu \mathrm{M})$ and $\mathrm{W} 7(50 \mu \mathrm{M})$ on the percentage of sperm showing (a) capacitation ( $B$ pattern) and (b) acrosome reacted ( $A R$ pattern) after chlortetracycline fluorescence (CTC) staining in media containing $\mathrm{CaCl}_{2}$ or in medium devoid of $\mathrm{CaCl}_{2}$ at $4 \mathrm{~h}$ of incubation period Results are expressed mean \pm standard error $(n=3)$. * Significantly different $(P<0.01)$ from all other treatments.

However, examination of the percentage of viable sperm indicated that the antagonist-induced inhibition was not due to nonspecific toxic effects (data not shown). These results indicate that CaM antagonists inhibit capacitation dependent membrane changes, and suggest that this pathway involves CaM. CaM can also make use of the calcium stores in the endoplasmic reticulum, and the sarcoplasmic reticulum. CaM undergoes a conformational change upon binding to calcium, which enables it to bind to specific proteins for a specific response. CaM is present in both the acrosomal region and the flagellum of sperm (Leclerc and Goupil, 2000), supporting the idea that $\mathrm{CaM}$ is in an appropriate location to regulate sperm membrane changes important for fertilization, and thus suggests that CaM plays a role in bovine sperm capacitation. 
Changes in sperm cyclic nucleotide metabolism have been implicated in a variety of sperm functions, including the initiation and maintenance of motility (Yanagimachi et al., 1994), induction of the AR (Kopf and Gerton, 1991), and capacitation (Stein and Fraser, 1984). Since the mammalian sperm enzyme has been demonstrated to be regulated directly/indirectly by $\mathrm{Ca}^{2+}$ (Hyne and Garbers, 1979), CaM (Gross et al., 1987) and $\mathrm{NaHCO}_{3}$ (Visconti et al., 1990). $\mathrm{Ca}^{2+}$ and $\mathrm{NaHCO}_{3}$ also appear to be required for capacitation (Boatman and Robbins, 1991) and the induction of the AR (Spira and Breitbart, 1992). Moreover, changes in the activity of adenylyl cyclase and cAMP have been postulated in some species to play a role in capacitation (Yanagimachi et al., 1994).
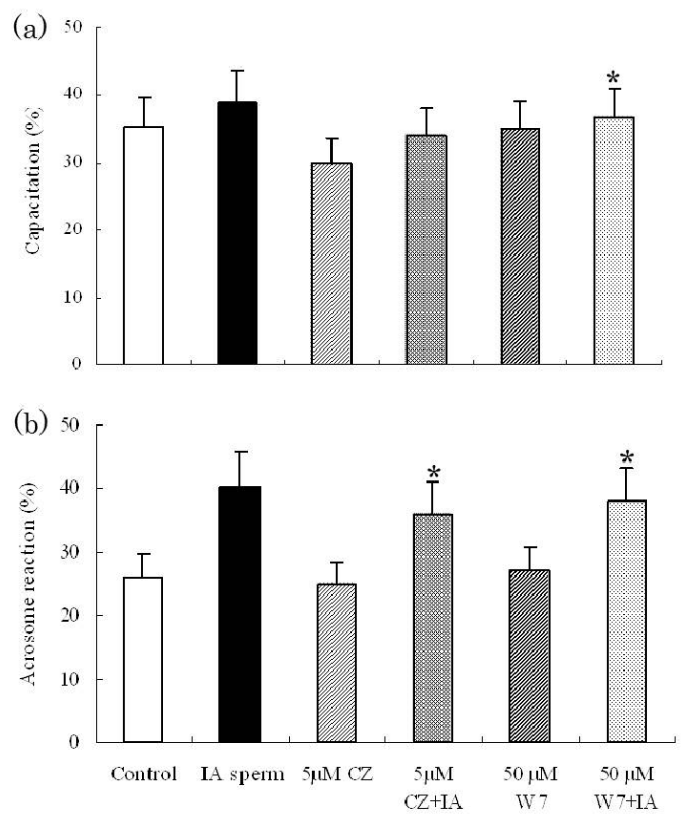

Figure 5. Effects of calcium ionophore $A 23187$ in presence of CaM antagonists $\mathrm{CZ}(5 \mu \mathrm{M})$ and W7 $(50 \mu \mathrm{M})$ on the percentage of sperm showing (a) capacitation (B pattern) and (b) acrosome reacted (AR pattern) after CTC staining at $4 \mathrm{~h}$ of incubation periods. Results are expressed mean \pm standard error $(n=3)$. * Significantly different $(\mathrm{P}<0.01)$ from the treatments where ionophore was not added with $\mathrm{CZ}$ and $\mathrm{W7}$.

In the study the effect of CaM antagonists ( $C Z$ and W7) on motility were not reversed by exogenous CAMP analogues. This finding is in agreement with a study of hamster sperm in which addition of IBMX to CZ-treated sperm increased the intracellular CAMP concentration to a level higher than that in capacitated sperm but did not restore hyperactivated motility [White and Aitken, 1989). Since both $\mathrm{Ca}^{2+}$ and $\mathrm{NaHCO}_{3}$ have been linked to capacitation and the regulation of mammalian sperm cAMP metabolism (Kopf and Gerton, 1991) and this intracellular second messenger has been implicated in both mammalian sperm capacitation and the $A R$, we were also interested in determining whether these membrane permeable cAMP analogues could support capacitation in CaM antagonists treated sperm in the absence of these ions. Figure 4 shows the experiments performed in media absence of $\mathrm{NaHCO}_{3}$. However, in the case of the $\mathrm{Ca}^{2+}$ depletion experiments, the membrane permeable CAMP analogues do not completely restore the $\mathrm{ZP}$-induced $A R$. This could be due to the fact that final stages of capacitation, presumably either independent of or downstream of the protein tyrosine phosphorylations, are not being completed, and/or $\mathrm{Ca}^{2+}$ is important components for the induction of the $A R$ (Yanagimachi et al., 1994).

The role of CAMP in the capacitation process as well as in the AR is still unknown (Aitken et al., 1998; Yanagimachi et al., 1994). It would be predicted that CAMP concentrations should rise during capacitation. Although some investigators have observed elevations in CAMP concentrations when spermatozoa were incubated under conditions that support capacitation (White and Aitken, 1989), others have reported decreasing concentrations of CAMP under these conditions (Stein and Fraser, 1984). In this study, when the sperm are incubated in the absence of $\mathrm{NaHCO}_{3}$, does not support capacitation. Regarding the possible participation of CAMP in the AR, it was observed in this present study that CAMP accumulation in $\mathrm{NaHCO}_{3}$-containing media was significantly higher than in $\mathrm{NaHCO}_{3}$-free medium. When the combination of dbcAMP and IBMX were added to $\mathrm{NaHCO}_{3}$-free media, the percentage of AR spermatozoa rose significantly, in comparison with the $\mathrm{AR}$ in the $\mathrm{NaHCO}_{3}$-containing medium. These results suggest that the ability of $\mathrm{NaHCO}_{3}$ to stimulate the $A R$ is mediated by a cAMP pathway. Using a biochemical approach for measuring the AR, Spira and Breitbart (Spira and Breitbart, 1992) reached similar conclusions in 
bovine spermatozoa, observing that $\mathrm{NaHCO}_{3}$ is no longer required when the CAMP levels are artificially elevated by the use of dbcAMP. The addition of dbcAMP and IBMX partially, but significantly, reversed the effect of CaM antagonists on the capacitation and AR. This study also supports the role of a cAMP dependent signaling pathway in capacitation (Aitken et al., 1998) via increased cAMP levels. Furthermore, these results reinforce the hypothesis that a loss of CaM accompanies capacitation of bovine sperm (Leclerc et al., 1992), thereby favoring the increased CAMP levels necessary to drive the signaling events associated with this phenomenon.

The calcium ionophore A23187 has been shown to induce the $A R$ in the sperm of almost all species tested, suggesting that $\mathrm{Ca}^{2+}$ is a primary mediator of this exocytotic reaction. Our results shows that CaM inhibitors have no observable effect on the calcium ionophore induced $A R$. When $C Z$ and $W 7$ were supplemented with ionophore, the percentage of AR spermatozoa significantly increased compared with the control and these antagonists treated sperm that were not supplemented with ionophore (Figure 6). The $A I$ induces the $A R$ by incorporating into the plasma membrane of sperm and transporting calcium, resulting in high intracellular calcium concentrations necessary for sperm capacitation and AR (Talbot et al., 1976).

\section{Conclusion}

The present study indicates that CaM is important for the control of bovine sperm motility and the inhibition of CaM antagonists on sperm motility might be due to uncontrolled influx of calcium ion of frozen-thawed bovine spermatozoa. The inhibition of CaM antagonists on capacitation and AR could partially overcome by supplementing CAMP analogues that were re-suspended in a capacitation supporting factors such as calcium and bicarbonate in the medium. These findings support the hypothesis that CaM antagonists can prevent capacitation and AR by interfering with multiple regulatory pathways. Thus, The results suggest that CaM may play an important role in the regulation of capacitation and $A R$ in frozenthawed bovine spermatozoa.

\section{References}

Aitken RJ, D Harkiss, W Knox, M Paterson and DS Irvine (1998). A novel signal transduction cascade in capacitating human spermatozoa characterised by a redox-regulated, CAMPmediated induction of tyrosine phosphorylation. Journal of Cell Science, 1115: 645-656.

Bendahmane M, C Lynch and DR Tulsiani (2001). Calmodulin signals capacitation and triggers the agonist-induced acrosome reaction in mouse spermatozoa. Archives of Biochemistry and Biophysics, 390: 1-8.

Boatman DE and RS Robbins (1991). Bicarbonate: carbon-dioxide regulation of sperm capacitation, hyperactivated motility, and acrosome reactions. Biology of Reproduction, 44: 806813.

Farlin ME, DJ Jasko, JK Graham and EL Squires (1992). Assessment of Pisum sativum agglutinin in identifying acrosomal damage in stallion spermatozoa. Molecular Reproduction and Development, 32: 23-27.

Fournier V, P Leclerc, N Cormier and JL Bailey (2003). Implication of calmodulin-dependent phosphodiesterase type 1 during bovine sperm capacitation. Journal of Andrology, 24: 104-112.

Gross MK, DG Toscano and WA Toscano (1987). Calmodulin-mediated adenylate cyclase from mammalian sperm. The Journal of Biological Chemistry, 262: 8672-8676.

Handrow RR, NL First and JJ Parrish (1989). Calcium requirement and increased association with bovine sperm during capacitation by heparin. Journal of Experimental Zoology, 252: 174-182.

Harayama H (2013). Roles of Intracellular Cyclic AMP Signal Transduction in the Capacitation and Subsequent Hyperactivation of Mouse and Boar Spermatozoa. Journal of Reproduction and Development, 59: 421-430.

Hook SS and AR Means (2001). $\mathrm{Ca}^{2+} / \mathrm{CaM}$-dependent kinases: from activation to function. Annual Review of Pharmacology and Toxicology, 41: 471-505.

Hyne RV and DL Garbers (1979). Calcium-dependent increase in adenosine $3^{\prime}, 5^{\prime}$-monophosphate and induction of the acrosome reaction in guinea pig spermatozoa. Proceedings of the National Academy of Sciences, 76: 5699-5703.

Kopf GS and GL Gerton (1991). The mammalian sperm acrosome and the acrosome reaction. In: Wassarman PM, (eds). Elements of Mammalian Fertilization. Boca. Raton, Fla: CRC Press, 153203.

Kuo YW., SH Li, K Maeda, B Gadella and PSJ Tsai (2016). The involvement of reproductive tract in sperm membrane surface modifications. Journal of Reproduction and Development, 25: 337-343.

Leclerc P and S Goupil (2000). Distribution and localization of calmodulin-binding proteins in bovine spermatozoa. Biology of Reproduction, 62: $1875-1881$. 
Leclerc P, MA Sirard, JG Chafouleas and RD Lambert (1992). Decrease in calmodulin concentrations during heparin-induced capacitation in bovine spermatozoa. Journal of Reproduction \& Infertility, 94: 23-32.

Mons N, JL Guillou and R Jaffard (1999). The role of $\mathrm{Ca}^{2+} /$ calmodulinstimulable adenylyl cyclases as molecular coincidence detectors in memory formation. Cellular and Molecular Life Sciences, 55: 525-533.

Moriya M, C Katagiri and K Yagi (1993). Immunoelectron microscopic localization of calmodulin and calmodulin-binding proteins in the mouse germ cells during spermatogenesis and maturation. Cell and Tissue Research, 271: 441451.

Rusnak F and P Mertz (2000). Calcineurin: form and function. Physiological Reviews, 80: 1483-1521.

Si $Y$ and $P$ Olds-Clarke (2000). Evidence for the involvement of calmodulin in mouse sperm capacitation. Biology of Reproduction, 62: 12311239.

White DR and RJ Aitken (1989). Relationship between calcium, cyclic AMP, ATP, and intracellular $\mathrm{pH}$ and the capacity of hamster spermatozoa to express hyperactivated motility. Gamete Research, 22: 163-77.

Spira B and H Breitbart (1992). The role of anion channels in the mechanism of acrosome reaction in bovine spermatozoa. Biochimica et Biophysica Acta, 1109: 65-73.

Stein DM and LR Fraser (1984). Cyclic nucleotide metabolism in mouse epididymal spermatozoa during capacitation in vitro. Gamete research, 10: 283- 299.

Talbot P, Summers RG, Hylander BL, Keough EM, Franklin LE (1976). The role of calcium in the acrosome reaction: an analysis using ionophore A23187. Journal of Experimental Zoology, 198: 383-392.

Tanphaichitr N and C Hansen (1994). Production of motile acrosome-reacted mouse sperm with nanomolar concentration of calcium ionophore A23187. Molecular Reproduction and Development, 37: 326-334.

Tardif S, C Dube, S Chevalier and JL Bailey (2001). Capacitation is associated with tyrosine phosphorylation and tyrosine kinase-like activity of pig sperm proteins. Biology of Reproduction, 65: 784-792.

Triana LR, DF Babcock, SP Lorton, NL First and HA Lardy (1980). Release of acrosomal hyaluronidase follows increased membrane permeability to calcium in the presumptive capacitation sequence for spermatozoa of the bovine and other mammalian species. Biology of Reproduction, 23: 47-59.

Visconti PE, L Johnson and M Oyaski (1997). Regulation, localization, and anchoring of protein kinase A subunits during mouse sperm capacitation. Developmental Biology, 192: 351363.

Visconti PE, GD Moore and JL Bailey (1995). Capacitation of mouse spermatozoa. II. Protein tyrosine phosphorylation and capacitation are regulated by a cAMPdependent pathway. Development, 121: 1139-1150.

Visconti PE, JP Muschietti, MM Flawia and JG Tezon (1990). Bicarbonate dependence of CAMP accumulation induced by phorbol esters in hamster spermatozoa. Biochimica et Biophysica Acta, 1054: 231-236.

Ward CR and BT Storey (1994). Determination of the time course of capacitation in mouse spermatozoa using a chlortetracycline fluorescence assay. Developmental Biology, 104: 287-296.

Wasco WM and GA Orr (1984). Function of calmodulin in mammalian sperm: presence of a calmodulin-dependent cyclic nucleotide phosphodiesterase associated with demembranated rat caudal epididymal sperm. Biochemical and Biophysical Research Communications, 118: 636-642.

Yanagimachi R (1994). Mammalian fertilization. In: Knobil E, Neill JD, edn. The Physiology of Reproduction, $2^{\text {nd }}$ ed. Raven Press, New York. pp. 189-317.

Zeng HT and DR Tulsiani (2003). Calmodulin antagonists differentially affect capacitationassociated protein tyrosine phosphorylation of mouse sperm components. Journal of Cell Science, 116: 1981-1989. 